\title{
EJERCICIO Y ALGUNOS MECANISMOS MOLECULARES QUE SUBYACEN A UNA MEJORA DEL DESEMPEÑO EN TAREAS COGNITIVAS
}

\section{EXERCISE AND SOME MOLECULAR MECHANISMS THAT UNDERLIE PERFORMANCE INCREASE ON COGNITIVE TASKS}

Bryan Montero-Herrera ${ }^{1 A B C D}$ y Jaime Fornaguera-Trías ${ }^{2 A B D}$

${ }^{1}$ Bryan Montero-Herrera. Profesor Escuela de Educación Física y Deportes Universidad de Costa Rica. Costa Rica. bryan_mh2005@hotmail.com

2 Jaime Fornaguera-Trías. Director Centro de Investigación en Neurociencias, Universidad de Costa Rica. Profesor Departamento de Bioquímica, Escuela de Medicina, Universidad de Costa Rica. Costa Rica. jaime.fornaguera@ucr.ac.cr

Responsabilidades.

ADiseño de la investigación

${ }^{\mathrm{B} R e d a c t o r}$ del trabajo

Cldea original y coordinador de toda la investigación

DBúsqueda de la literatura

Recibido el 24 de noviembre de 2019

Aceptado el 12 de enero de 2020

Correspondencia: Bryan Montero-Herrera: bryan_mh2005@hotmail.com

DOI: http://dx.doi.org/10.24310/riccafd.2020.v9i1.8303

\section{RESUMEN}

Potenciar el aprendizaje y la memoria se ha convertido en una tarea de interés para muchos investigadores. Artículos recientes han podido comprobar que el ejercicio es una clara herramienta para mejorar capacidades cognitivas de personas y animales gracias a que favorece la liberación de sustancias tales como neurotransmisores y factores neurotróficos. Uno de los factores más importantes es el factor neurotrófico derivado del cerebro (BDNF), que promueve la generación de cambios a nivel neuronal necesarios para la potenciación a largo plazo (fortalecimiento de memorias). Se realizó una búsqueda en las bases de datos de EBSCOhost -en las bases de Academic Search Complete, ERIC, MEDLINE, PsycARTICLES y SPORTDiscus-, Google Scholar, Pubmed y ScienceDirect utilizando una serie de palabras con sus respectivos criterios de inclusión y exclusión. En este trabajo se explican los procesos asociados con la potenciación a largo plazo, abarcando temas como neuroplasticidad, la unión del BDNF con su receptor (receptores de tirosina quinasa $\mathrm{B}$ ), y además el mecanismo por medio del cual la liberación de la mioquina irisina, como una consecuencia del ejercicio, promueve el aumento de 
las concentraciones de BDNF en sangre. Basándose en numerosa literatura se describe la relación que existe entre el ejercicio y la generación de cambios neuroplásticos estimulados por proteínas como el BDNF y la consecuente mejoría en la memoria.

Palabras clave: aprendizaje, BDNF, ejercicio, irisina, memoria, potenciación a largo plazo.

\section{ABSTRACT}

Increase learning and memory has been one of the major goals for many researchers. Recent articles have proved that exercise can influence some cognitive capacities in people and animals. These benefits are obtained for releasing substances such as neurotransmitters and neurotrophic factors. One of the most important factors is the brain derived neurotrophic factor (BDNF), which promotes changes in neurons necessary for long term potentiation (memory strengthening). A search was carried out in the databases EBSCOhost -in the bases de Aca-demic Search Complete, ERIC, MEDLINE, PsycARTICLES y SPORTDiscus-, Google Scholar, Pubmed and ScienceDirect using words with their respective inclusion and exclusion criteria. In this work is explained the processes related with long term potentiation, including topics such as: neuroplasticity, join of BDNF with its receptor (tyrosine kinase receptor). Moreover, some aspects about the mechanisms by means of which the myokine irisin, after exercise promotes an increase of BDNF concentrations in blood. Based in many literature it describes the relation between exercise and neuroplastic changes induced by proteins such as BDNF, and the final improvement in memory.

Key words: learning, BDNF, exercise, irisin, memory, long term potentiation.

\section{INTRODUCCIÓN}

Los beneficios que la práctica del ejercicio tiene en las personas que lo practican, han sido bien documentados desde hace mucho tiempo (1). Estos beneficios se han logrado detectar incluso en procesos cognitivos complejos como el aprendizaje y la memoria (2-5).

Tanto el aprendizaje -tarea que permite la adquisición de la información que se encuentra alrededor- como la memoria -que permite la codificación, almacenamiento, consolidación y recuperación de esa información que fue previamente aprendida- son procesos que involucran diversas regiones del cerebro como el hipocampo y la corteza cerebral (6-8). En el hipocampo, la realización de ejer-cicio aeróbico (principalmente) y de contra resistencia, incrementa la liberación de la proteína llamada factor neurotrófico derivado del cerebro (BDNF, por sus siglas en inglés) que se unirá posteriormente a sus receptores conocidos como tirosina quinasa $B$ (TrkB, por sus siglas en inglés) $(9,10)$. 
La unión del BDNF con su receptor TrkB forman parte de los mecanismos que subyacen a las mejoras que se observan en el aprendizaje y la memoria como consecuencia del ejercicio (2,11-14). El aprendizaje y la memoria se asocian con cambios cerebrales que incluyen generalmente modificaciones en el número de células nerviosas y el fortalecimiento de las sinapsis, que son las encargadas de asegurar la comuni-cación entre ellas. A estos procesos se les conoce genéricamente como neuroplasticidad (15-17). La neuroplasticidad se define como la capacidad del sistema nervioso para responder a cambios en el ambiente externo o interno, que en última instancia le permitirán al organismo "enfrentarse con su medio" de la mejor manera posible (15). A nivel celular uno de los mecanismos más estudiados con relación a la neuroplasticidad y que subyace a la memoria es la potenciación a largo plazo (PLP) (18-20). Tanto la realización de ejercicio como los consecuentes aumentos de BDNF, propician que los cambios a nivel neuronal sean más eficientes y duraderos, lo que resulta en la mejora de la PLP $(13,21-23)$.

El objetivo de este trabajo consiste en describir y analizar de manera general evidencia sobre cómo la liberación del BDNF inducida por el ejercicio, conduce a la generación de PLP y como consecuencia a una mejoría en la memoria.

\section{METODOLOGÍA}

La recopilación de información se efectuó entre los meses de marzo y diciembre del año 2018 en los buscadores EBSCOhost y en las bases de Academic Search Complete, ERIC, MEDLINE, PsycARTICLES y SPORTDiscus-, Google Scholar, Pubmed y ScienceDirect. Las palabras claves utilizadas en cada uno de los buscadores se combinaron con un único descriptor tanto en el idioma español ("y") como en el idioma inglés ("and"), las mismas fueron: "ejercicio y BDNF", "ejercicio y BDNF y potenciación a largo plazo", "ejercicio e irisina y BDNF", "ejercicio e irisina y BDNF y potenciación a largo plazo", "exercise and BDNF", "exercise and BDNF and long term potentiation", "exercise and BDNF and long term potentiation", "exercise and irisin and BDNF", "exercise and irisin and BDNF and long term potentiation".

El proceso para seleccionar cada una de las investigaciones fue: planteamiento de diversos criterios de inclusión y exclusión para escoger los diseños experimentales y no experimentales que estuvieran más acordes con las variables ejercicio, irisina, BDNF y potenciación a largo plazo. Luego se continuó con la lectura de cada uno de los resúmenes ("abstracts") para conocer si la información estaba relacionada con la temática a desarrollar en el presente trabajo. En caso de que lo estuviera, se leía el texto completo para verificar las variables, el diseño y los procedimientos para así determinar la inclusión del mismo a la muestra final. Es a partir de todo este proceso que se fueron elaborando cada uno de los subapartados que conforman la revisión.

Se incluyeron los estudios que cumplieran con los siguientes criterios: idioma español o inglés, estudios en humanos, ratas o ratones, que existiera 
una relación o efecto entre el ejercicio y alguna de las siguientes variables: irisina, BDNF y potenciación a largo plazo ya sea de manera individual (e.g.: aumentos de la irisina después de ejercitarse) o por medio de la combinación de varias (e.g.: aumento de la irisina y el BDNF central después de ejercitarse) ya sea en artículos experimentales, cuasiexperimentales o revisiones de literatura sobre artículos científicos. Los criterios de exclusión fueron: no incluir al ejercicio como variable independiente, no tener mediciones pre y post cuando analizaban alguna prueba cognitiva o del BDNF, tener algún incentivo económico o cualquier otro tipo cuando los participantes resolvían las pruebas cognitivas. Todas las investigaciones que cumplieran con los criterios de inclusión fueron descargados, la identificación de la duplicación de los artículos se efectuó manualmente.

\section{NEUROPLASTICIDAD}

Durante el desarrollo del individuo ocurren cambios en todos los sistemas fisiológicos como parte del proceso de maduración. Se forman nuevas células, se empiezan a definir cada uno de los órganos que le permitirán al individuo vivir en concordancia con su ambiente. El sistema nervioso no es la excepción, y en este proceso aumenta su tamaño y se empiezan a producir conexiones entre células que llevarán la información de un lado al otro de nuestro cuerpo de forma rápida y precisa. En cada una de las etapas del neurodesarrollo se dan cambios en el ambiente que nos rodea, aparecen nuevos estímulos, como la luz, los olores, los sabores que empiezan a moldear de alguna manera las conexiones que se establecen entre las diferentes células y las diferentes regiones cerebrales. Esto se conoce cómo neuroplasticidad, un término que en los últimos tiempos se ha utilizado en muchísimos contextos para explicar entre otros los cambios en el comportamiento y en el desarrollo de las funciones cognitivas $(16,18-23)$.

La neuroplasticidad involucra una serie de procesos tales como: la sinaptogénesis (crecimiento de dendritas o axones que permiten aumentar 0 hacer nuevas conexiones con otras células), el desenmascaramiento (activación de conexiones que en un principio estaban inhibidas), síntesis de sinapsinas (encargadas de anclar las vesículas sinápticas al citoesqueleto), liberación de neurotransmisores (por exocitosis de vesículas situadas en las terminales presinápticas) y finalmente la PLP (que son cambios electrofisiológicos que perduran por periodos de tiempo prolongados después de una estimulación repetida) (24). En este artículo nos enfocaremos principalmente en esta última.

\section{POTENCIACIÓN A LARGO PLAZO (PLP)}

Es una forma de plasticidad que permite un incremento en la transmisión sináptica por un período prolongado de tiempo $(25,26)$. La PLP es generada por estímulos eléctricos cortos pero de una alta frecuencia (tetanización) provenientes de la célula presináptica y que permanecen activos en el tiempo, despolarizando en mayor medida a las células postsinápticas (27-29). Uno de 
los aspectos más elevantes de la PLP es su especificidad o también llamada marca sináptica (30) que se caracteriza porque solo las vías neurales que muestran la tetania son las que tienen mejoría, mientras que otras vías sinápticas adyacentes no las presentan.

La PLP es un proceso, que por sus características (duración del estímulo, frecuencia del estímulo y los cambios que se producen a nivel celular) puede dividirse en dos etapas, una de ellas es la potenciación a largo plazo temprana (PLP-T) que se ha asociado con la memoria a corto plazo y la segunda sería la potenciación a largo plazo tardía (PLP-TA) que se ha dicho subyace a la memoria a largo plazo (26).

La PLP-T empieza inmediatamente después de estímulos que generen un PLP, es decir se induce con una seguidilla de estímulos de alta frecuencia. Además, es independiente de la síntesis proteica y eso también la diferencia de la PLP-TA $(6,27,31)$.

Esta PLP-T inicia con la liberación de glutamato (principal neurotransmisor excitatorio) de las células presinápticas, el cual se encuentra almacenado en vesículas sinápticas (vGLUT1). Las moléculas de glutamato atraviesan la hendidura sináptica y se unen a sus receptores del tipo AMPA y NMDA en la célula postsináptica. Los receptores AMPA permiten la entrada de sodio $\left(\mathrm{Na}^{+}\right)$ mientras que los NMDA favorecen la entrada de calcio $\left(\mathrm{Ca}^{2+}\right)$.

Al entrar el $\mathrm{Na}+$ a través de los receptores AMPA, se produce una despolarización de la célula postsináptica que favorece la remoción del ion $\mathrm{Mg}^{2+}$ que se encuentra bloqueando los receptores NMDA. Al eliminarse este bloqueo ingresa $\mathrm{Ca}^{2+}$ a la célula, que una vez dentro de la célula tiene varios efectos como lo es la activación de la Proteínkinasa C (PKC) y/o la Kinasa II calcio Calmodulina dependiente (CaMKII) las cuales se encargan de movilizar un contingente adicional de receptores AMPA hacia la membrana celular. Al aumentar la concentración de estos receptores en la membrana celular aumenta consecuentemente la interacción del glutamato con ellos y por lo tanto aumentan las concentraciones de $\mathrm{Na}^{+}$intracelular lo que mantendrá los receptores NMDA por más tiempo abiertos. Simultáneamente la PKC y la CaMKII liberan óxido nítrico (NO), ácido araquidónico (AA) y factor de agregación plaquetaria (FAP) (32), los cuales pueden desplazarse hasta la célula presináptica y ahí aumentar la liberación de glutamato, completando el ciclo de activación (Ver figura 1).

Por su parte la PLP-TA requiere de tres o cuatro seguidillas de estimulación espaciadas. Puede mantenerse por más de cuatro horas, inclusive días, semanas o años (33). La PLP-TA activa diferentes vías de transducción, involucrando entre otros un aumento en la concentración de calcio intracelular. Activa la vía de CREB, que es un factor de transcripción que a su vez favorece la expresión de algunos genes y finalmente la síntesis de nuevas proteínas, que cumplen diversas funciones, entre las que se encuentran promover la neuro-plasticidad (34). 

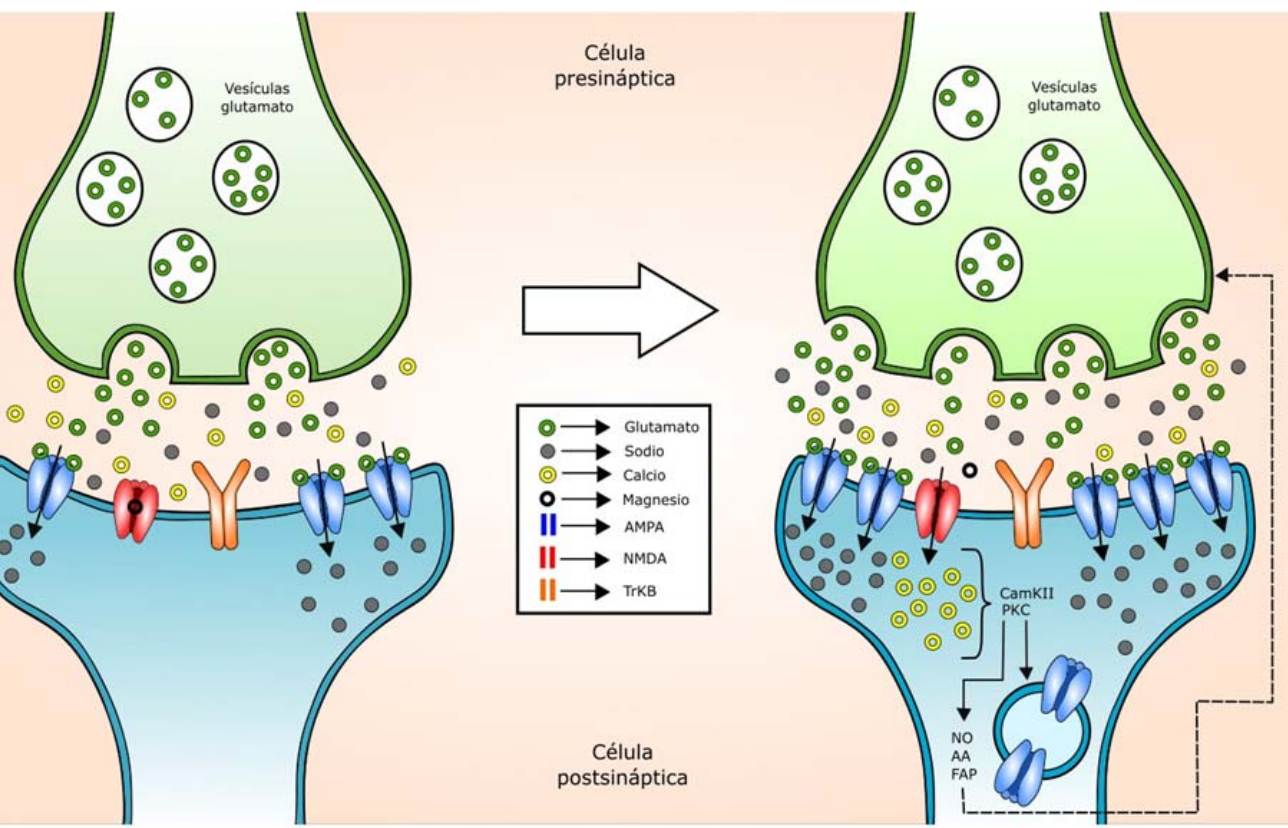

(-) $\odot \circ \circ, 0$
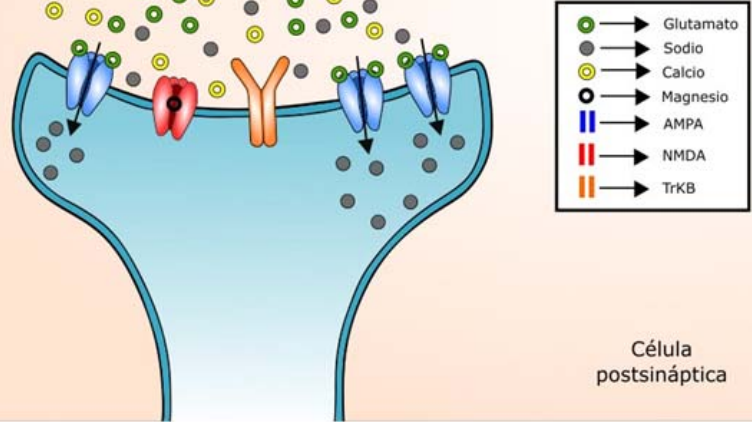

Figura 1. PLP-T Izq.: Liberación de los neurotransmisores de glutamato de sus vesículas sinápticas por medio de la exocitosis. Dos moléculas de glutamato se adhieren a cada uno de los receptores AMPA permitiendo la entrada de sodio. Der: Los cambios de concentración de sodio en el interior de la célula postsináptica provocan que se retire el magnesio que bloquea los receptores de NMDA, permitiendo así la entrada de calcio, lo cual activa a las proteínas CamKII y PKC que indirectamente, por procesos de fosforilación, favorecen la movilización e inserción de los receptores AMPA en la membrana. Además, en este proceso se libera óxido nítrico (NO), ácido araquidónico (AA) y factor de agregación plaquetaria (FAP) que actúan como mensajeros retrógrados en la célula presináptica, resultando en un mantenimiento de la activación de la sinapsis (retrocontrol positivo). Elaborada por Mónica Vargas San Gil.

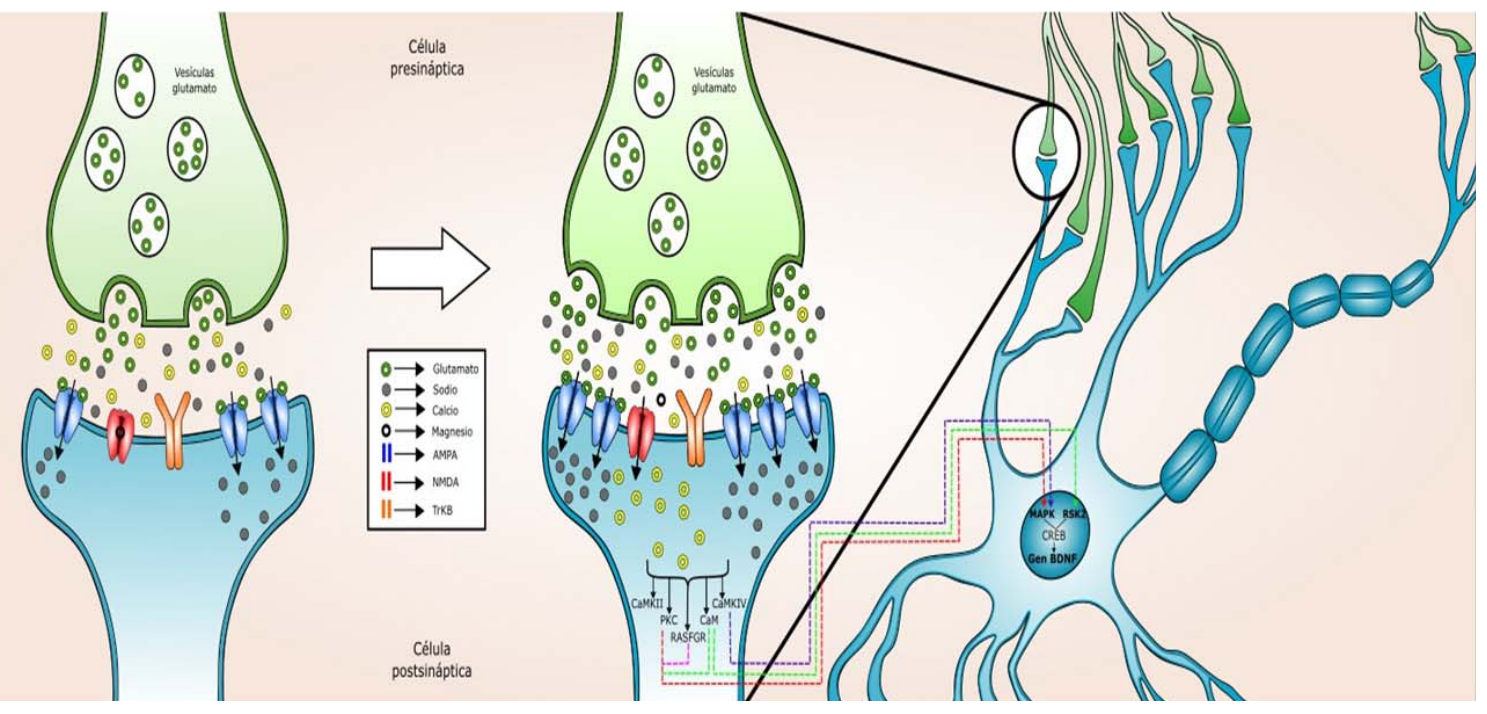

Figura 2. PLP-TA Izq.: Liberación de los neurotransmisores de glutamato de sus vesículas sinápticas y consecuente unión de dos glutamatos a los receptores AMPA que permiten la entrada de sodio. Der.: Apertura de los receptores NMDA por el cambio en la concentración interna de sodio en la célula, esto provoca una entrada masiva de calcio que activa cascadas moleculares (PKC, RASFGR, CaM y CaMIV) a nivel del citoplasma y sus señalizaciones llegarán al núcleo teniendo un efecto sobre MAPK y RSK2 que activarán a CREB. Una vez CREB empieza a funcionar se activa la expresión de algunos genes entre ellos el gen BDNF que pasará a convertirse en el factor neurotrófico derivado del cerebro (BDNF). Elaborada por Mónica Vargas San Gil. 


\section{¿QUÉ ES EL BDNF?}

Es un péptido que pertenece a la familia de las neurotrofinas cuya función principal es promover el crecimiento y la supervivencia de las neuronas, tanto a nivel del sistema nervioso central, como del sistema nervioso periférico (35). En los años 50 se identificó el primer factor neurotrófico, que recibió el nombre de factor de crecimiento neural (NGF, por sus siglas en inglés) (36). Ya para el año 1982 se descubrió otro factor en el cerebro, que cumplía las funciones de las neurotrofinas y al que llamaron Factor Neurotrófico Derivado del Cerebro (BDNF por sus siglas en inglés) (37). Posteriormente se observó que durante el ejercicio se da un aumento en los niveles periféricos de BDNF (36) que concuerdan con un incremento en los niveles de esta neurotrofina en el Sistema Nervioso Central también durante el ejercicio (38).

El BDNF actúa en varias regiones cerebrales tales como el hipocampo, la amígdala, la corteza cerebral, el hipotálamo, el área tegmental ventral, el núcleo del tracto solitario y la sustancia nigra (39-41). Este péptido ha sido relacionado con una gran diversidad de procesos, entre ellos el control de la dirección y la velocidad de crecimiento de las espinas dendríticas $(42,43)$, otros procesos de plasticidad sináptica como la neurogénesis $(44,45)$, y funcionalmente en el aprendizaje, la memoria $(11,46)$ y la PLP $(47,48)$.

\section{INTERACCIÓN BDNF-RECEPTORES TRKB}

Todas las neurotrofinas se sintetizan en forma de una proteína precursora (prepro-neurotrofina), que, a través de proteólisis o ruptura de enlaces, se va modificando estructuralmente. El ejercicio puede activar la vía de CREB, que entre otras favorece la síntesis de diferentes ARNm que se traducirán luego en proteínas. El BDNF es sintetizado como una prepro-neurotrofina y almacenado como tal en las vesículas. Una vez allí es transformado por proteólisis en el proBDNF, que podría permanecer dentro de la célula o ser liberado como pro-BDNF o en su forma madura el mBDNF, que también se genera por proteólisis. El proBDNF se une a los receptores p75NTR mientras que el mBDNF se une al TrkB $(15,49-52)$. Las funciones que cada una de estas neurotrofinas desencadena al unirse con su receptor son diferentes, pero siempre relacionadas con procesos de neuroplasticidad (Ver figura 2).

Dependiendo del tipo de BDNF que se haya liberado se producirá una cascada de reacciones que promueven o inhiben la PLP-TA. Cuando el proBDNF se une con el receptor p75NTR el resultado final es la apoptosis, depresión sináptica y una reducción de la dendrogénesis (53-55). En contraste cuando es el mBDNF el que se une a sus receptores TrkB, estos fosforilan varias enzimas como la fosfatidil inositol 3 kinasa ( $P I 3 K$, por sus siglas en inglés), las MAPK y la fosfolipasa C (PLCr, por sus siglas en inglés), que tienen como uno de sus efectos principales la liberación de más BDNF (53-55) (Ver figura 2). 
El BDNF que se libera de la célula postsináptica tiene dos opciones: actuar en esa misma célula o viajar a la célula presináptica. En la célula presináptica, se sugiere que aumenta el número de vesículas que se encontrarán en la zona activa, prestas a liberar neurotransmisores como el glutamato $(56,57)$.

En la célula postsináptica, por su lado, la activación del receptor de TrkB incrementa la acción de los receptores AMPA (aumentando su exposición a nivel de la membrana celular) y también los de NMDA (que permitirán un mayor entrada de $\mathrm{Ca}^{2+}$ ). Como se mencionó anteriormente, tanto el aumento en los receptores AMPA, como también el aumento en los niveles intracelulares de calcio (a través de los receptores NMDA) cumplen un papel fundamental en la generación de la PLP (58-60). Existe también evidencia de que el BDNF aumenta en la membrana la cantidad de receptores de TrkB, la supervivencia y el crecimiento celular, así como la plasticidad sináptica en zonas como el hipocampo y la corteza cerebral $(51,61,62)$ (Ver figura 3$)$.

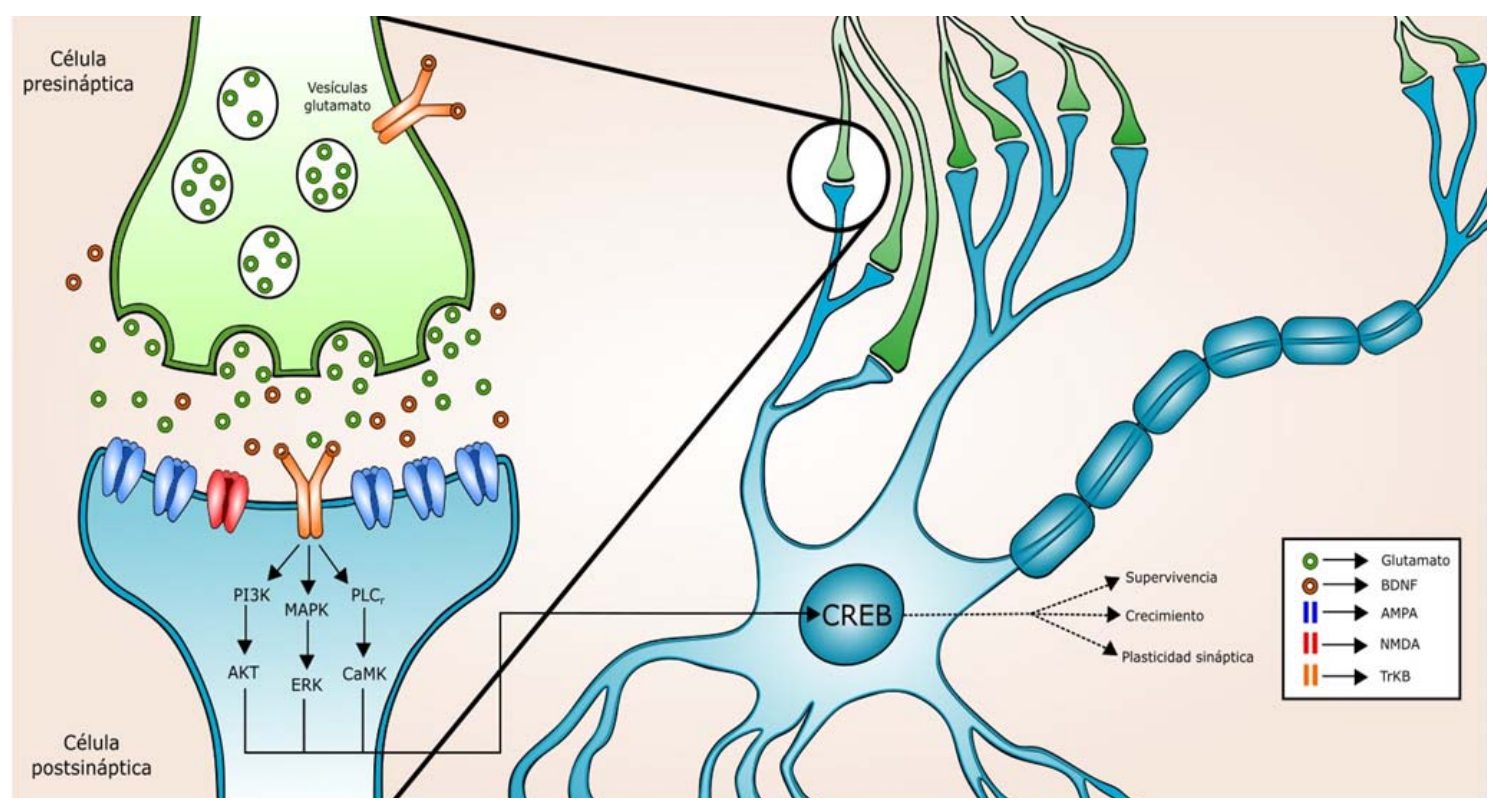

Figura 3. Una vez que el BDNF ha sido liberado de la célula postsináptica este puede viajar, unirse y activar receptores de TrkB tanto en la célula presináptica como en la postsináptica. En la célula presináptica aumentará la liberación del neurotransmisor glutamato. En la célula postsináptica, cuando el BDNF se acopla a su receptor de TrkB activará una serie de cascadas moleculares (PI3K, MAPK, PLCr) que provocan a nivel del citoplasma activar de igual forma AKT, ERK y CaMK. Son estas últimas las encargadas de activar en el núcleo a CREB y así provocar que se lleven a cabo procesos de supervivencia, crecimiento y plasticidad sináptica. Elaborada por Mónica Vargas San Gil.

\section{¿CÓMO EL EJERCICIO AUMENTA LA EXPRESIÓN DE BDNF?}

Hay bastante literatura en la que se demuestra que después del ejercicio las concentraciones de BDNF en el sistema nervioso central son mayores en grupos de animales ejercitados cuando se comparan con animales que no hicieron ejercicio $(44,46,49)$. Estos aumentos se asocian con mejoras en procesos tales como neurogénesis, aprendizaje, memoria y $\operatorname{PLP}(20,48)$. 
Los aumentos en el BDNF parecen ser el producto de fenómenos que ocurren durante el ejercicio tales como: un aumento de la actividad simpática, un aumento en los niveles de noradrenalina, un aumento del factor de crecimiento asociado a la insulina 1, y por cambios en los estados energéticos o de la disponibilidad de oxígeno (58,63-65). Además, cuando el músculo se ejercita, ha logrado demostrarse que libera hormonas llamadas mioquinas, como la irisina, que se sabe aumenta la expresión de BDNF tanto en el área tegmental ventral, como también en el hipocampo (66-69).

Durante el ejercicio, el músculo aumenta la producción del proliferador de peroxisoma, activador del receptor $y$ coactivador $1 \alpha-$ (PGC-1a, por sus siglas en inglés), el cual actúa como un activador transcripcional regulando procesos como el metabolismo energético y la liberación de factores del músculo $(70,71)$. La unión del PGC-1a con los receptores de estrógeno alfa de humano (ERRa), en el tejido muscular activan así la expresión del gen Fndc5, posteriormente Ilevando a la síntesis de la proteína fibronectina tipo III con 5 dominios (FNDC5, por sus siglas en inglés). Esta proteína sufre una modificación postraduccional y forma la mioquina llamada irisina a nivel muscular (72-74).

Luego de formada, la irisina pasará al torrente sanguíneo donde será transportada y llegará eventualmente a la barrera hematoencefálica $(66,67,69)$. Es justamente cuando la irisina llega a esta barrera donde existe controversia en la literatura, ya que no se sabe si logra traspasarla o si más bien activa algún otro factor que se encarga de generar los efectos a nivel central (67-69). Ha sido propuesto igualmente a través de una serie de experimentos que cuando el BDNF es liberado a nivel central este puede disminuir la aparición del gen Fndc5, que por consiguiente no formará el FNDC5 y por lo tanto no se sintetizará la irisina (75) (Ver figura 4).

\section{RELACIÓN ENTRE EJERCICIO, BDNF, TRKB Y PLP EN LOS PROCESOS DE MEMORIA Y APRENDIZAJE}

En un estudio (76) en el que se utilizaron ratas como sujeto experimental se investigó la relación existente entre el ejercicio, el BDNF y la mejora de ciertos procesos cognitivos. Para ello bloquearon los receptores de TrkB inyectando una sustancia (TrkB-IgG) a nivel del hipocampo justo antes de realizar el ejercicio. Una vez finalizado el tratamiento se realizó una prueba en el Laberinto de Morris (con el que se mide adquisición y retención de la memoria espacial) y se analizaron las concentraciones del ARNm de BDNF, TrkB, y CREB en todo el hipocampo (no especifican una zona en particular) por medio de la técnica Reacción en Cadena de la Polimerasa en tiempo real (RT-PCR).

Las ratas se mantuvieron en cajas individualmente durante todo el experimento. Se distribuyeron en cuatro grupos (grupo de ratas sedentarias (SCC), ratas con ejercicio (ECC) inyectadas intracerebralmente con citocromo $\mathrm{C}$ (es una condición control, no tiene efectos sobre ningún receptor), el grupo tres y cuatro 
estaban conformados por ratas sedentarias (STrkB) y que habían hecho ejercicio (ETrkB) inyectadas con TrkB-lgG intracerebralmente) (76). Recibieron una inyección solo en el lado derecho del hipocampo.

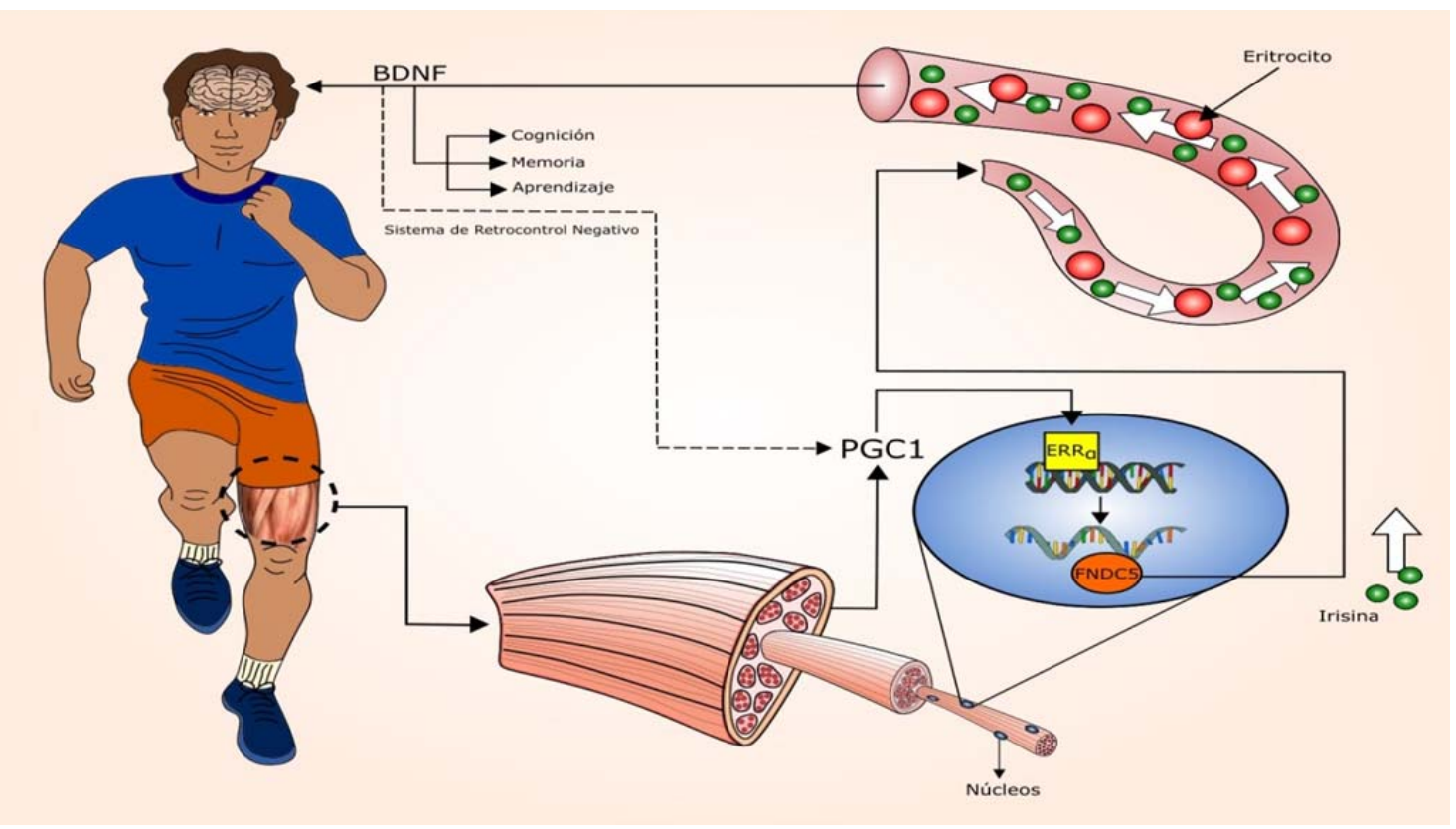

Figura 4. El ejercicio aeróbico aumenta la liberación de PGC1a que se acopla con sus receptores ERRa para activar a FNDC5 la cual forma la irisina y esta es enviada al torrente sanguíneo donde a través de unos mecanismos aumenta la liberación de BDNF a nivel del cerebro. El BDNF no solo se ha asociado con procesos de cognición, aprendizaje y memoria, sino que también sirve como sistema de retrocontrol negativo para disminuir la producción deFNDC5 (línea punteada). Elaborada por Mónica Vargas San Gil.

El protocolo consistió en un entrenamiento en el laberinto de Morris por 5 días con 2 pruebas por día (medir adquisición), descansaron 2 días y se les volvió a someter al mismo laberinto para medir la retención, para lo cual quitaron la plataforma de donde estuvo durante el periodo de adquisición (cuadrante P) y midieron el tiempo que los animales nadaban en cada cuadrante del laberinto.

Los resultados demostraron que la latencia (tiempo para hallar la plataforma) en los dos primeros días disminuyó y fue muy similar entre los 4 grupos. Sin embargo, el grupo de ECC presentó latencias significativamente menores que las ratas del grupo sedentario (SCC) en los días tres, cuatro, cinco y en la sesión posterior a los 2 días (retención). Además, los investigadores hallaron en el grupo ECC incrementos significativos en los niveles de ARNm de BDNF, TrkB y CREB en el hipocampo que se sugiere podrían estar asociados con las diferencias conductuales observadas.

El grupo con los receptores de TrkB bloqueados (ETrkB) mostró un aumento significativo en el tiempo de latencia al compararlos con el grupo SCC. Entre los grupos sedentarios de cada condición (STrkB y SCC) no se presentaron diferencias. Los grupos ETrkB, STrkB y SCC presentaron valores menores que el grupo ECC en cuanto al ARNm de BDNF, TrkB y CREB. 
En otro estudio (77) analizaron la relación del ejercicio aeróbico, con el BDNF, el TrkB y la PLP utilizando ratones Ts65Dn macho con Síndrome de Down (SD) y un grupo control de ratones silvestres (WT, por sus siglas en inglés). Fueron asignados 2 animales por caja a una condición sedentaria y a otra experimental (rueda de ejercicio donde accedían de forma voluntaria) por 4 semanas. Se realizaron varias pruebas conductuales, entre ellas la prueba de Localización Espacial del Objeto (OL, por sus siglas en inglés) y la prueba de Localización del Objeto Nuevo (NOR, por sus siglas en inglés) en las cuales permanecieron por 15 minutos en 3 días diferentes. Además, se midió la actividad motora en cada una de esas pruebas, calculando la distancia recorrida que el ratón hizo dentro de la caja al finalizar el tratamiento.

Para la prueba OL, cuando comparan la condición sedentarios y de ejercicio tanto para los WT como los SD, se encontró que los ratones que realizaron ejercicio permanecen significativamente por más tiempo investigando el objeto que fue movido 24 horas después. Pero al aplicar la prueba en la condición de ejercicio de ambos grupos, se observó que el rendimiento fue similar entre ellos. Se sugiere, basados en estos resultados que los animales SD ejercitados tuvieron la memoria espacial activa por más tiempo que sus pares sedentarios. Igualmente se mostró que el tiempo invertido para analizar el objeto movido fue significativamente mayor en el grupo SD que realizó ejercicio en comparación con el grupo SD sedentario. Ambos resultados apuntan a que independiente-mente de la condición del ratón WT o SD el ejercicio favorece procesos de me-moria en esta tarea específica.

Con el test NOR, igualmente los animales sedentarios con SD fueron significativamente peores en discriminar el objeto novedoso en comparación con los WT. En cambio, en la condición ejercicio ambos grupos aumentaron su capacidad discriminativa, pero las diferencias entre ellos no fueron significativas. El ejercicio parece entonces revertir los efectos de la condición SD. Cuando se realizó la comparación entre grupos, observaron que el grupo WT y SD que se ejercitaban, tuvieron mejores desempeños que los grupos sedentarios.

En este estudio los investigadores además del análisis conductual, estudiaron diferentes fenómenos que subyacen a la plasticidad neuronal en el hipocampo (e.g.: potenciales postsinápticos, vesículas de neurotransmisores) y demostraron una asociación importante entre el ejercicio y la expresión del ARNm y de la proteína de BDNF, que promueve una plasticidad estructural y funcional de las neuronas de esta área cerebral, lo cual se asocia además con un mejor rendimiento en pruebas de memoria.

Estudios realizados con humanos han tratado de explicar en cierta manera los hallazgos en modelos animales (78-81) haciendo mediciones de BDNF en sangre (i.e., arteria yugular, arteria braquial, vena yugular y vena cubital) tanto antes como después de una sesión de ejercicio. Obtienen que los niveles del BDNF aumentan hasta un $60 \%$ de su estado basal y permanecen incrementados 30 minutos y hasta 60 minutos después 
de finalizado el ejercicio. En una investigación (78) los participantes asistieron en varias ocasiones al laboratorio para ejercitarse por 30 minutos a intensidades diferentes. Finalizado el ejercicio y 24 horas después se les aplicó la prueba de memoria Test de Aprendizaje Auditivo Verbal de Rey (RAVLT, por sus siglas en inglés) la cual consiste en una lista con 15 palabras que la persona escucha y repite al final. La lista A se leyó 5 veces y fue repetida en 5 ocasiones. Posterior a eso, se leyó una lista $\mathrm{B}$ que el participante repitió una vez y después tuvieron que volver a decir las palabras de la lista A. Para finalizar, hicieron una pausa de 30 minutos en silencio o leyendo y luego dijeron nuevamente la lista A. Transcurridas 24 horas, los llamaron por teléfono y se les leyó de manera aleatoria las palabras de cada una de las listas y 20 palabras distractoras, el objetivo era que los sujetos reconocieran si dicha palabra formaba parte de la lista $A$, de la $B$ o de ninguna. Además, se les extrajeron muestras de sangre para medir los niveles de BDNF antes del ejercicio, inmediatamente post ejercicio y a los 30 minutos post ejercicio.

Los resultados mostraron que el desempeño en la lista A durante las 5 oca-siones mejoró significativamente del ensayo 1 al 2 y del 2 al 3, pero del ensayo 3 al 4 y del 4 al 5 estas diferencias no se presentaron. La prueba efectuada a los 30 minutos post ejercicio no presentó diferencias significativas. La medición a las 24 horas demostró que a mayor intensidad del ejercicio el resultado de la prueba fue más alto. Los niveles de BDNF fueron mayores cuando la intensidad era más elevada, pero no lograron hallar diferencias o relaciones entre el BDNF y las pruebas de memoria.

En otro estudio (9), reclutaron a 40 sujetos a los cuales dividieron en cuatro grupos: tres de ejercicio aeróbico (baja, moderada y alta intensidad) y uno control (estiramiento). El tratamiento duró 12 semanas, asistiendo a cuatro sesiones por semana con una duración de 30 minutos cada una. Tanto el primer día como en el último día del tratamiento a los sujetos les extrajeron sangre de la vena mediana cubital para medir las concentraciones de BDNF. Además, les aplicaron la Escala de Inteligencia de Wechsler para Niños-IV (WISC-IV, por sus siglas en inglés) para medir la capacidad de memoria de trabajo. Esta prueba se compone de varios subtests, de los que los autores escogieron el de tipo números. El mismo consiste en listas diferentes que deberán decirse en el mismo orden o inverso. La primera hilera de ambos tests inició con dos números, cada vez que se repitió de la forma correcta se le agregó un número más, hasta completar un total de 8 dígitos. Se comenzó con la prueba que los números se repiten en el mismo orden, una vez finalizada se hizo un descanso de dos minutos y luego hicieron otra. Cuando ambas listas se completaron pasaron a realizar la otra condición (orden inverso) en dos ocasiones.

Después de las 12 semanas los niveles de BDNF en sangre en el grupo de alta y moderada intensidad fueron más altos en estado basal en comparación con su primera medición. Además, el grupo de alta intensidad fue significativamente diferente del grupo de baja intensidad y el control. En el caso del grupo de moderada intensidad, fue significativamente diferente del grupo control. Los 
resultados en la prueba de memoria sugieren que ejercitarse a una alta intensidad durante un período de 12 semanas mejora más el desempeño en la prueba Wechsler que las otras 3 condiciones experimentales.

Muestras de irisina en humanos después de una sesión de ejercicio también han sido obtenidas por medio de extracciones de sangre de la vena cubital (82) y de la vena basílica (83). En la investigación de Murawska-Cialowicz (83) se incluyeron 12 mujeres y 12 hombres quienes participaron en el mismo tratamiento. La medición inicial se realizó en dos momentos diferentes. En la primera semana, les tomaron muestras de sangre para medir las concentraciones de BDNF e irisina, luego realizaron la Prueba de Wingate (i.e., pedalear por 30 segundos a máxima intensidad en un cicloergómetro) y finalizada la prueba les midieron nuevamente BDNF e irisina. La siguiente semana, lo único que varió fue el test físico, ya que en vez del de Wingate hicieron un Test progresivo de consumo máximo de Oxígeno (cada 3 minutos se aumentan la carga de trabajo 50 Watts mientras la persona pedaleaba). Posteriormente participaron en 3 meses de un entrenamiento de CrossFit (i.e., consistía en ejercicios aeróbicos y contra resistencia) al cual asistieron a dos sesiones a la semana de una duración de 60 minutos cada uno. Transcurridos los 3 meses volvieron aplicar las mismas primeras pruebas en el mismo orden.

Los resultados demostraron que los niveles de BDNF a los tres meses fueron significativamente más elevados en mujeres y en hombres en comparación con la primera medición en las variables de reposo y con la prueba de Wingate. Con lo que respecta a la prueba progresiva, tanto en hombres como en mujeres disminuyeron la concentración de los valores de BDNF al comparar la primera con la última medición. La concentración de irisina, en las mediciones de reposo al inicio del tratamiento estuvo más elevada en mujeres que hombres, sin embargo, al final de los tres meses más bien se observó una disminución significativa, y en el caso de los hombres aumentó, pero no hubo diferencias ni entre la primera medición ni con ninguna de las mediciones de las mujeres. En la prueba de Wingate y en la progresiva no hubo diferencias entre las variables género y medición.

\section{CONCLUSIONES}

El ejercicio puede ser visto como un agente que promueve la generación de cambios neuroplásticos debido al impacto que tiene en la expresión de proteínas como el BDNF, que ha sido frecuentemente asociada con el crecimiento, supervivencia neuronal y con la plasticidad sináptica. Una forma de aumentar los niveles de este factor es aumentando la actividad metabólica muscular, lo que incrementa la liberación de proteínas como la irisina, que a su vez promueve la expresión del ARNm del BDNF y de la síntesis de la proteína correspondiente, a través de mecanismos complejos que se están empezando a dilucidar. En los estudios en humanos no se realizaron análisis simultáneos de BDNF, irisina y memoria, por lo que establecer una asociación directa entre los tres factores es en este momento aventurado.

\section{EJERCICIO Y ALGUNOS MECANISMOS MOLECULARES QUE SUBYACEN A UNA MEJORA DEL DESEMPEÑO EN TAREAS COGNITIVAS}


Se han utilizado diversas estrategias experimentales tanto, en seres humanos, como en modelos animales para tratar de analizar dicha asociación. Entre ellas está la práctica de ejercicio y la medición de BDNF tanto a nivel plasmático o cerebral, y también se han hecho experimentos donde se realiza ejercicio y al mismo tiempo se inactivan los receptores de TrkB para BDNF. Los resultados soportan la hipótesis de que el ejercicio favorece procesos cognitivos como la memoria por medio de cambios en la PLP. Pero si se hace ejercicio y al mismo tiempo se ven inactivados los receptores TrkB, estos efectos no serán mayores a los del grupo control.

Es importante señalar, sin embargo, que hay que hacer una lectura crítica de la literatura que aborda los efectos del ejercicio sobre la conducta y las funciones cognitivas. Es imperativo analizar las posibles diferencias que podrían obtenerse dependiendo del tipo de ejercicio (ej.: aeróbico, anaeróbico, contra resistencia), de la etapa del desarrollo en la que se realiza (etapas tempranas, pubertad, edad adulta), de la duración de esos ejercicios (crónico, agudo), de su intensidad, del sexo y por supuesto de la condición de salud del sujeto. Solo tomando en cuenta estas variables y quizás otras más tendremos un panorama más realista, que nos permita entonces comprender los mecanismos subyacentes a las diferentes condiciones experimentales, y así poder concluir al respecto de la relación que existe entre el desempeño en tareas cognitivas particulares y los diferentes tipos de ejercicio.

AGRADECIMIENTOS: A Mónica Vargas San Gil por la elaboración de las ilustraciones que se presentan en este trabajo.

\section{REFERENCIAS BIBLIOGRÁFICAS}

1. Bird SR, Smith A, James K. Exercise Benefits and Prescription. Nelson Thornes; 1998. 348 p.

2. Berchtold NC, Castello N, Cotman CW. Exercise and time-dependent benefits to learning and memory. Neuroscience. mayo de 2010;167(3):588-97.

3. Fordyce DE, Wehner JM. Physical activity enhances spatial learning performance with an associated alteration in hippocampal protein kinase $\mathrm{C}$ activity in C57BL/6 and DBA/2 mice. Brain Res. agosto de 1993;619(1-2):111-9.

4. Hall JM, Savage LM. Exercise leads to the re-emergence of the cholinergic/ nestin neuronal phenotype within the medial septum/diagonal band and subsequent rescue of both hippocampal ACh efflux and spatial behavior. Exp Neurol. abril de 2016;278:62-75.

5. Kramer AF, Hahn S, Cohen NJ, Banich MT, McAuley E, Harrison CR, et al. Ageing, fitness and neurocognitive function. Nature. 29 de julio de 1999;400(6743):418-9.

6. Ortega Loubon C, César Franco J. Neurofisiología del aprendizaje y la memoria. Plasticidad Neuronal. Arch Med. 2010;6(7):1-7. 
7. Poolton JM, Masters RSW, Maxwell JP. The relationship between initial errorless learning conditions and subsequent performance. Hum Mov Sci. junio de 2005;24(3):362-78.

8. Reber PJ. The neural basis of implicit learning and memory: A review of neuropsychological and neuroimaging research. Neuropsychologia. agosto de 2013;51(10):2026-42.

9. Jeon $\mathrm{YK}, \mathrm{Ha} \mathrm{CH}$. The effect of exercise intensity on brain derived neurotrophic factor and memory in adolescents. Environ Health Prev Med. diciembre de 2017;22(1):27.

10.Tsai C-L, Chen F-C, Pan C-Y, Wang C-H, Huang T-H, Chen T-C. Impact of acute aerobic exercise and cardiorespiratory fitness on visuospatial attention performance and serum BDNF levels. Psychoneuroendocrinology. marzo de 2014;41:121-31.

11.Cunha C, Brambilla R, Thomas K. A simple role for BDNF in learning and memory? Front Mol Neurosci. 2010;3:1-14.

12.Erickson KI, Voss MW, Prakash RS, Basak C, Szabo A, Chaddock L, et al. Exercise training increases size of hippocampus and improves memory. Proc Natl Acad Sci U S A. 108(7):3017-22.

13.Farmer J, Zhao X, van Praag H, Wodtke K, Gage F., Christie B. Effects of voluntary exercise on synaptic plasticity and gene expression in the dentate gyrus of adult male sprague-dawley rats in vivo. Neuroscience. enero de 2004;124(1):71-9.

14.Griffin ÉW, Mullally S, Foley C, Warmington SA, O’Mara SM, Kelly ÁM. Aerobic exercise improves hippocampal function and increases BDNF in the serum of young adult males. Physiol Behav. 24 de octubre de 2011;104(5):934-41.

15.Ballesteros JJ. El papel del sistema BDNF-TrkB en la plasticidad sináptica independiente de receptores tipo NMDA inducida por xantinas [Tesis de doctorado en Neurociencias]. [Departamento de Fisiología]: Universidad Autónoma de Madrid; 2015.

16.Kandel ER. The Molecular Biology of Memory Storage: A Dialogue Between Genes and Synapses. Science. 2 de noviembre de 2001;294(5544):1030-8.

17. Martin SJ, Grimwood PD, Morris RGM. Synaptic Plasticity and Memory: An Evaluation of the Hypothesis. Annu Rev Neurosci. marzo de 2000;23(1):649-711.

18. Gruart A. Involvement of the CA3-CA1 Synapse in the Acquisition of Associative Learning in Behaving Mice. J Neurosci. 25 de enero de 2006;26(4):1077-87.

19. Gruart A, Sciarretta C, Valenzuela-Harrington M, Delgado-Garcia JM, Minichiello L. Mutation at the TrkB PLC -docking site affects hippocampal LTP and associative learning in conscious mice. Learn Mem. 3 de enero de 2007;14(12):54-62.

20.Nabavi S, Fox R, Proulx CD, Lin JY, Tsien RY, Malinow R. Engineering a memory with LTD and LTP. Nature. julio de 2014;511(7509):348-52. 
21.Dietrich MO, Mantese CE, Porciuncula LO, Ghisleni G, Vinade L, Souza DO, et al. Exercise affects glutamate receptors in postsynaptic densities from cortical mice brain. Brain Res. diciembre de 2005; 1065(1-2):20-5.

22.Shimizu E. NMDA Receptor-Dependent Synaptic Reinforcement as a Crucial Process for Memory Consolidation. Science. 10 de noviembre de 2000; 290(5494):1170-4.

23. Yu Q, Li X, Wang J, Li Y. Effect of exercise training on long-term potentiation and NMDA receptor channels in rats with cerebral infarction. Exp Ther Med. diciembre de 2013;6(6):1431-6.

24.Aguilar F. Plasticidad cerebral. Parte 1. Rev Med IMSS. 2003;4(1):55-60.

25.Bliss TVP, Cooke SF. Long-term potentiation and long-term depression: a clinical perspective. Clinics. 2011;66:3-17.

26. Huang EP. Synaptic plasticity: going through phases with LTP. Curr Biol CB. 7 de mayo de 1998;8(10):R350-352.

27.Soderling TR, Derkach VA. Postsynaptic protein phosphorylation and LTP. Trends Neurosci. febrero de 2000;23(2):75-80.

28.Leff P, Romo H, Matus M, Hernández A, Calva JC, Acevedo R, et al. Understanding the neurobiological mechanisms of learning and memory: memory systems of the brain, long term potentiation and synaptic plasticity part III. Salud Ment. 2002;25(4):78-94.

29.Nicoll R. A Brief History of Long-Term Potentiation. Neuron. 2017; 93(2): 281-290.

30.López Rojas J, Almaguer Melián W, Bergado Rosado JA. La 'marca sináptica' y la huella de la memoria. Rev Neurol. 2007;45(10):607-14.

31.Frey S, Bergado-Rosado J, Seidenbecher T, Pape H-C, Frey JU. Reinforcement of Early Long-Term Potentiation (Early-LTP) in Dentate Gyrus by Stimulation of the Basolateral Amygdala: Heterosynaptic Induction Mechanisms of Late-LTP. J Neurosci. 15 de mayo de 2001; 21(10):3697-703.

32. Maureira F. Plasticidad sináptica, bdnf y ejercicio físico. Rev Digit Educ Física. 2016; 7(40):51-63.

33.Adams JP, Dudek SM. Late-phase long-term potentiation: getting to the nucleus. Nat Rev Neurosci. septiembre de 2005;6(9):737-43.

34.Alvarado B. Participación del óxido nítrico y la horma arginina vasopresina en el aprendizaje y la memoria [Tesis de maestría en Ciencias Fisiológicas]. [Colombia]: Universidad de Colima; 2006.

35.Piepmeier AT, Etnier JL. Brain-derived neurotrophic factor (BDNF) as a potential mechanism of the effects of acute exercise on cognitive performance. Journal of Sport and Health Science. marzo de 2015; 4(1): 14-23.

36. Levi-Montalcini R, Hamburger V. Selective growth stimulating effects of mouse sarcoma on the sensory and sympathetic nervous system of the chick embryo. J Exp Zool. marzo de 1951;116(2):321-61. 
37. Barde YA, Edgar D, Thoenen $\mathrm{H}$. Purification of a new neurotrophic factor from mammalian brain. EMBO J. mayo de 1982;1(5):549-53.

38. Karege F, Schwald M, Cisse M. Postnatal developmental profile of brain-derived neurotrophic factor in rat brain and platelets. Neurosci Lett. agosto de 2002;328(3):261-4.

39.Conner JM, Lauterborn JC, Yan Q, Gall CM, Varon S. Distribution of BrainDerived Neurotrophic Factor (BDNF) Protein and mRNA in the Normal Adult Rat CNS: Evidence for Anterograde Axonal Transport. J Neurosci. 1 de abril de 1997; 17(7):2295-313.

40.Cotman C. Exercise: a behavioral intervention to enhance brain health and plasticity. Junio de 2002. Trends in Neurosciences; 25(6): 295-301.

41.Vanevski F, Xu B. Molecular and neural bases underlying roles of BDNF in the control of body weight. Front Neurosci. 2013;7.

42.Ji Y, Pang PT, Feng L, Lu B. Cyclic AMP controls BDNF-induced TrkB phosphorylation and dendritic spine formation in mature hippocampal neurons. Nat Neurosci. febrero de 2005;8(2):164-72.

43.Kwon M, Fernandez JR, Zegarek GF, Lo SB, Firestein BL. BDNF-Promoted Increases in Proximal Dendrites Occur via CREB-Dependent Transcriptional Regulation of Cypin. J Neurosci. 29 de junio de 2011;31(26):9735-45.

44.Liu PZ, Nusslock R. Exercise-Mediated Neurogenesis in the Hippocampus via BDNF. Front Neurosci. 7 de febrero de 2018;12:52.

45.Ramírez-Rodríguez G, Ocaña-Fernández MA, Vega-Rivera NM, Torres-Pérez OM, Gómez-Sánchez A, Estrada-Camarena E, et al. Environmental enrichment induces neuroplastic changes in middle age female BalbC mice and increases the hippocampal levels of BDNF, p-Akt and p-MAPK1/2. Neuroscience. febrero de 2014;260:158-70.

46.Sheikhzadeh F, Etemad A, Khoshghadam S, AsI NA, Zare P. Hippocampal BDNF content in response to short- and long-term exercise. Neurol Sci. julio de 2015;36(7):1163-6.

47.Lu Y, Christian K, Lu B. BDNF: A key regulator for protein synthesis-dependent LTP and long-term memory? Neurobiol Learn Mem. marzo de 2008;89(3):31223.

48.Panja D, Bramham CR. BDNF mechanisms in late LTP formation: A synthesis and breakdown. Neuropharmacology. enero de 2014;76:664-76.

49.Galeano HP. El papel del ejercicio físico en la inducción de BDNF y sus vías de señalización en el sistema nervioso central. Aplicación neurobiológica en modelos sanos y terapéutica en la enfermedad de Alzheimer. [Tesis de doctorado en Fisiología]. [Departamento de Fisiología]: Universidad de Valencia; 2014.

50.Lessmann V, Gottmann K, Malcangio M. Neurotrophin secretion: current facts and future prospects. Prog Neurobiol. abril de 2003;69(5):341-74.

91 EJERCICIO Y ALGUNOS MECANISMOS MOLECULARES QUE SUBYACEN A UNA MEJORA DEL DESEMPEÑO EN TAREAS COGNITIVAS 
51.Adachi N. New insight in expression, transport, and secretion of brain-derived neurotrophic factor: Implications in brain-related diseases. World J Biol Chem. 2014; 5(4):409.

52. Hökfelt T, Broberger C, Xu Z-QD, Sergeyev V, Ubink R, Diez M. Neuropeptides — an overview. Neuropharmacology. julio de 2000;39(8):1337-56.

53.Koshimizu H, Kiyosue K, Hara T, Hazama S, Suzuki S, Uegaki K, et al. Multiple functions of precursor BDNF to CNS neurons: negative regulation of neurite growth, spine formation and cell survival. Mol Brain. 2009; 2(1):27.

54.Lee R. Regulation of Cell Survival by Secreted Proneurotrophins. Science. 30 de noviembre de 2001;294(5548):1945-8.

55.Lu B, Chang JH. Regulation of neurogenesis by neurotrophins: implications in hippocampus-dependent memory. Neuron Glia Biol. 20 de julio de 2005;1(04):377.

56. Jovanovic JN, Czernik AJ, Fienberg AA, Greengard P, Sihra TS. Synapsins as mediators of BDNF-enhanced neurotransmitter release. Nat Neurosci. abril de 2000; 3(4):323-9.

57.Tyler WJ, Pozzo-Miller LD. BDNF enhances quantal neurotransmitter release and increases the number of docked vesicles at the active zones of hippocampal excitatory synapses. J Neurosci Off J Soc Neurosci. 15 de junio de 2001; 21(12):4249-58.

58.Ding Q, Vaynman S, Akhavan M, Ying Z, Gomez-Pinilla F. Insulin-like growth factor I interfaces with brain-derived neurotrophic factor-mediated synaptic plasticity to modulate aspects of exercise-induced cognitive function. Neuroscience. enero de 2006;140(3):823-33.

59.Itami C, Kimura F, Kohno T, Matsuoka M, Ichikawa M, Tsumoto T, et al. Brainderived neurotrophic factor-dependent unmasking of «silent» synapses in the developing mouse barrel cortex. Proc Natl Acad Sci. 28 de octubre de 2003; 100(22):13069-74.

60.Narisawa-Saito M, Iwakura Y, Kawamura M, Araki K, Kozaki S, Takei N, et al. Brain-derived Neurotrophic Factor Regulates Surface Expression of a-Amino3-hydroxy-5-methyl-4-isoxazoleproprionic Acid Receptors by Enhancing the $\mathrm{N}$-Ethylmaleimide-sensitive Factor/GluR2 Interaction in Developing Neocortical Neurons. J Biol Chem. 25 de octubre de 2002; 277(43):40901-10.

61. Haapasalo A, Sipola I, Larsson K, Åkerman KEO, Stoilov P, Stamm S, et al. Regulation of TRKB Surface Expression by Brain-derived Neurotrophic Factor and Truncated TRKB Isoforms. J Biol Chem. 8 de noviembre de 2002; 277(45):43160-7.

62. Huang EJ, Reichardt LF. Neurotrophins: Roles in Neuronal Development and Function. Annu Rev Neurosci. marzo de 2001;24(1):677-736.

63.Arany Z, Foo S-Y, Ma Y, Ruas JL, Bommi-Reddy A, Girnun G, et al. HIF-independent regulation of VEGF and angiogenesis by the transcriptional coactivator PGC-1a. Nature. febrero de 2008;451(7181):1008-12. 
64.Garcia C, Chen M., Garza A., Cotman C., Russo-Neustadt A. The influence of specific noradrenergic and serotonergic lesions on the expression of hippocampal brain-derived neurotrophic factor transcripts following voluntary physical activity. Neuroscience. julio de 2003;119(3):721-32.

65.West AE, Greenberg ME. Neuronal Activity-Regulated Gene Transcription in Synapse Development and Cognitive Function. Cold Spring HarbPerspect Biol. 1 de junio de 2011; 3(6):a005744-a005744.

66. Erickson HP. Irisin and FNDC5 in retrospect: An exercise hormone or a transmembrane receptor? Adipocyte. 22 de octubre de 2013; 2(4):289-93.

67.Islam MR, Young MF, Wrann CD. The Role of FNDC5/Irisin in the Nervous System and as a Mediator for Beneficial Effects of Exercise on the Brain. En: Spiegelman B, editor. Hormones, Metabolism and the Benefits of Exercise [Internet]. Cham: Springer International Publishing; 2017 [citado 7 de abril de 2019]. p. 93-102. (Research and Perspectives in Endocrine Interactions).

68.Panati K, Suneetha Y, Narala VR. Irisin/FNDC5--An updated review. Eur Rev Med Pharmacol Sci. 2016;20(4):689-97.

69.Papp C, Pak K, Erdei T, Juhasz B, Seres I, Szentpéteri A, et al. Alteration of the irisin-brain-derived neurotrophic factor axis contributes to disturbance of mood in COPD patients. Int J Chron Obstruct Pulmon Dis. 7 de julio de 2017;12:2023-33.

70.Boström P, Wu J, Jedrychowski MP, Korde A, Ye L, Lo JC, et al. A PGC1- dependent myokine that drives brown-fat-like development of white fat and thermogenesis. Nature. 11 de enero de 2012;481(7382):463-8.

71.Jin $\mathrm{Y}$, Sumsuzzman DM, Choi J, Kang H, Lee S-R, Hong Y. Molecular and Functional Interaction of the Myokine Irisin with Physical Exercise and Alzheimer's Disease. Molecules [Internet]. 7 de diciembre de 2018 [citado 7 de abril de 2019];23(12).

72.Wrann CD, White JP, Salogiannnis J, Laznik-Bogoslavski D, Wu J, Ma D, et al. Exercise Induces Hippocampal BDNF through a PGC-1a/FNDC5 Pathway. Cell Metab. noviembre de 2013;18(5):649-59.

73.Xu B. BDNF (I)rising from Exercise. Cell Metab. noviembre de 2013;18(5):612-4.

74.Zhorne R, Dudley-Javoroski S, Shields R. Skeletal muscle activity and CNS neuroplasticity. Neural Regen Res. 2016;11(1):69.

75. Mattson MP. Evolutionary aspects of human exercise-Born to run purposefully. Ageing Res Rev. julio de 2012;11(3):347-52.

76.Vaynman S, Ying Z, Gomez-Pinilla F. Hippocampal BDNF mediates the efficacy of exercise on synaptic plasticity and cognition. Eur J Neurosci. noviembre de 2004;20(10):2580-90.

77.Parrini M, Ghezzi D, Deidda G, Medrihan L, Castroflorio E, Alberti M, et al. Aerobic exercise and a BDNF-mimetic therapy rescue learning and memory in a mouse model of Down syndrome. Sci Rep. diciembre de 2017;7(1):16825.

93 EJERCICIO Y ALGUNOS MECANISMOS MOLECULARES QUE SUBYACEN A UNA MEJORA DEL DESEMPEÑO EN TAREAS COGNITIVAS 
78.EtnierJL, Wideman L, Labban JD, Piepmeier AT, Pendleton DM, Dvorak KK, Becofsky $\mathrm{K}$. The effects of acute exercise on memory and brain-derived neurotrophic factor (BDNF). Journal of Sport and Exercise Psychology. junio 8 de 2016; 38(4): 331-340.

79.Rasmussen P, Brassard P, Adser H, Pedersen MV, Leick L, Hart E, et al. Evidence for a release of brain $\square$ derived neurotrophic factor from the brain during exercise. Experimental physiology. octubre de 2009; 94(10): 1062-1069.

80.SeifertT, Brassard P, Wissenberg M, RasmussenP, NordbyP, Stallknecht B., et al. Endurance training enhances BDNF release from the human brain. American Journal of Physiology-Regulatory, Integrative and Comparative Physiology. noviembre 18 de 2009; 298(2):R372-7.

81.Walsh JJ, Tschakovsky, ME. Exercise and circulating BDNF: mechanisms of release and implications for the design of exercise interventions. Applied Physiology, Nutrition, and Metabolism. noviembre de 2018; 43(11): 1095-1104.

82.Benedini S, Dozio E, Invernizzi PL, Vianello E, Banfi G, Terruzzi I, et al. Irisin: a potential link between physical exercise and metabolism-an observational study in differently trained subjects, from elite athletes to sedentary people. Journal of Diabetes Research. 2017; 1-7.

83.Murawska-Cialowicz E, Wojna J, Zuwala-Jagiello J. Crossfit training changes brain-derived neurotrophic factor and irisin levels at rest, after wingate and progressive tests, and improves aerobic capacity and body composition of young physically active men and women. J Physiol Pharmacol. diciembre de 2015; 66(6): 811-821. 